\title{
The enumeration of coverings of closed orientable Euclidean manifolds $\mathcal{G}_{3}$ and $\mathcal{G}_{5}$.
}

\author{
G. Chelnokov* \\ National Research University Higher School of Economics \\ grishabenruven@yandex.ru \\ A. Mednykh ${ }^{\dagger}$ \\ Sobolev Institute of Mathematics, \\ Novosibirsk, Russia \\ Novosibirsk State University, \\ Novosibirsk, Russia \\ mednykh@math.nsc.ru
}

\begin{abstract}
There are only 10 Euclidean forms, that is flat closed three dimensional manifolds: six are orientable and four are non-orientable. The aim of this paper is to describe all types of $n$-fold coverings over orientable Euclidean manifolds $\mathcal{G}_{3}$ and $\mathcal{G}_{5}$, and calculate the numbers of non-equivalent coverings of each type. We classify subgroups in the fundamental groups $\pi_{1}\left(\mathcal{G}_{3}\right)$ and $\pi_{1}\left(\mathcal{G}_{5}\right)$ up to isomorphism and calculate the numbers of conjugated classes of each type of subgroups for index $n$. The manifolds $\mathcal{G}_{3}$ and $\mathcal{G}_{5}$ are uniquely determined among the others orientable forms by their homology groups $H_{1}\left(\mathcal{G}_{3}\right)=\mathbb{Z}_{3} \times \mathbb{Z}$ and $H_{1}\left(\mathcal{G}_{5}\right)=\mathbb{Z}$.

Key words: Euclidean form, platycosm, flat 3-manifold, non-equivalent coverings, crystallographic group.
\end{abstract}

2010 Mathematics Subject Classification: 20H15, 57M10, 55R10.

\section{Introduction}

Let $\mathcal{M}$ be a manifold with fundamental group $\Gamma=\pi_{1}(\mathcal{M})$. Two coverings

$$
p_{1}: \mathcal{M}_{1} \rightarrow \mathcal{M} \text { and } p_{2}: \mathcal{M}_{2} \rightarrow \mathcal{M}
$$

\footnotetext{
*This work was supported by the Russian Foundation for Basic Research (grant 18 - 01 - 00036/18).

$\dagger$ This work was supported by the Russian Foundation for Basic Research (grant 16-31-00138).
} 
are said to be equivalent if there exists a homeomorphism $h: \mathcal{M}_{1} \rightarrow \mathcal{M}_{2}$ such that $p_{1}=p_{2} \circ h$. According to the general theory of covering spaces, any $n$-fold covering is uniquely determined by a subgroup of index $n$ in the group $\Gamma$. The equivalence classes of $n$-fold covering of $\mathcal{M}$ are in one-to-one correspondence with the conjugacy classes of subgroups of index $n$ in the fundamental group $\pi_{1}(\mathcal{M})$. See, for example, ([7], p. 67). In such a way the following two natural problems arise. The first one is to calculate the number of subgroups of given finite index $n$ in $\pi_{1}(\mathcal{M})$. The second problem is to find the number of conjugacy classes of subgroups of index $n$ in $\pi_{1}(\mathcal{M})$.

The problem of enumeration for nonequivalent coverings over a Riemann surface with given branch type goes back to the paper [8] by Hurwitz, in which the number of coverings over the Riemann sphere with given number of simple (of order two) branching points was determined. Later, in [9], it has been proved that this number can be expressed in the terms of irreducible characters of symmetric groups. The Hurwitz problem was studed by many authors. A detailed survey of the related results is contained in ([13], [10]). For closed Riemann surfaces, this problem was completely solved in [16]. However, of most interest is the case of unramified coverings. Let $s_{\Gamma}(n)$ denote the number of subgroups of index $n$ in the group $\Gamma$, and let $c_{\Gamma}(n)$ be the number of conjugacy classes of such subgroups. According to what was said above, $c_{\Gamma}(n)$ coincides with the number of nonequivalent $n$-fold coverings over a manifold $\mathcal{M}$ with fundamental group $\Gamma$. The numbers $s_{\Gamma}(n)$ and $c_{\Gamma}(n)$ for the fundamental group of a closed surface (orientable or not) were found in (14], 15], 17]). In the paper [18, a general method for calculating the number $c_{\Gamma}(n)$ of conjugacy classes of subgroups in an arbitrary finitely generated group $\Gamma$ was given. Asymptotic formulas for $s_{\Gamma}(n)$ in many important cases were obtained by T. W. Müller and his collaborators ([19], [20], [21]).

In the three-dimensional case, for a large class of Seifert fibrations, the value of $s_{\Gamma}(n)$ was calculated in [11] and [12]. In our previous papers [1] and 2] the numbers $s_{\Gamma}(n)$ and $c_{\Gamma}(n)$ were determined for the fundamental group of non-orientable Euclidian manifolds $\mathcal{B}_{1}$ and $\mathcal{B}_{2}$ whose homologies are $H_{1}\left(\mathcal{B}_{1}\right)=\mathbb{Z}_{2} \oplus \mathbb{Z}^{2}$ and $H_{1}\left(\mathcal{B}_{2}\right)=\mathbb{Z}^{2}$ and for the orientable Euclidean manifolds $\mathcal{G}_{2}$ and $\mathcal{G}_{4}$ with $H_{1}\left(\mathcal{G}_{2}\right)=\mathbb{Z}_{2} \oplus \mathbb{Z}_{2} \oplus \mathbb{Z}$ and $H_{1}\left(\mathcal{G}_{4}\right)=\mathbb{Z}_{2} \oplus \mathbb{Z}$ respectively.

The aim of the present paper is to investigate $n$-fold coverings over orientable Euclidean three dimensional manifolds $\mathcal{G}_{3}$ and $\mathcal{G}_{5}$, with the first homologies $H_{1}\left(\mathcal{G}_{3}\right)=\mathbb{Z}_{3} \oplus \mathbb{Z}$ and $H_{1}\left(\mathcal{G}_{5}\right)=\mathbb{Z}$. We classify subgroups of finite index in the fundamental groups of $\pi_{1}\left(\mathcal{G}_{3}\right)$ and $\pi_{1}\left(\mathcal{G}_{5}\right)$ up to isomorphism. Then we calculate the number of subgroups and the number of conjugacy classes of subgroups of each isomorphism type for a given index $n$.

We note that numerical methods to solve these and similar problems for the threedimensional crystallogical groups were developed by the Bilbao group [4]. The first homologies of all the three-dimensional crystallogical groups are determined in [22].

\section{Notations}

We use the following notations: $s_{H, G}(n)$ is the number of subgroups of index $n$ in the group $G$, isomorphic to the group $H ; c_{H, G}(n)$ is the number conjugacy classes of sub- 
groups of index $n$ in the group $G$, isomorphic to the group $H$.

Also we will need the following combinatorial functions:

$$
\begin{gathered}
\sigma_{0}(n)=\sum_{k \mid n} 1, \quad \sigma_{1}(n)=\sum_{k \mid n} k, \quad \sigma_{2}(n)=\sum_{k \mid n} \sigma_{1}(k), \quad \omega(n)=\sum_{k \mid n} k \sigma_{1}(k), \\
\theta(n)=\left|\left\{(p, q) \mid p>0, q \geq 0, p^{2}-p q+q^{2}=n\right\}\right| .
\end{gathered}
$$

In all cases we consider the function vanished if $n \notin \mathbb{N}$.

Remark. It can be shown that $\theta(n)=\sum_{k \mid n}\left(\frac{k}{3}\right)$, where $\left(\frac{k}{3}\right)$ is the Legendre symbol, see [6] p.112. This representation clarifies the analogy between the functions $\sigma_{1}(n)$ and $\theta(n)$, and makes the appearance of the latter one less amazing. However, this representation will not be used further.

\section{Overview}

The main goal of this paper is to prove the following results.

The first theorem provides the complete solution of the problem of enumeration of subgroups of a given finite index in $\pi_{1}\left(\mathcal{G}_{3}\right)$.

Theorem 1. Every subgroup $\Delta$ of finite index $n$ in $\pi_{1}\left(\mathcal{G}_{3}\right)$ is isomorphic to either $\pi_{1}\left(\mathcal{G}_{3}\right)$ or $\pi_{1}\left(\mathcal{G}_{1}\right) \cong \mathbb{Z}^{3}$. The respective numbers of subgroups are

$$
\begin{gathered}
s_{\pi_{1}\left(\mathcal{G}_{3}\right), \pi_{1}\left(\mathcal{G}_{3}\right)}(n)=\sum_{k \mid n} k \theta(k)-\sum_{k \mid \frac{n}{3}} k \theta(k), \\
s_{\mathbb{Z}^{3}, \pi_{1}\left(\mathcal{G}_{3}\right)}(n)=\omega\left(\frac{n}{3}\right) .
\end{gathered}
$$

The next theorem provides the number of conjugacy classes of subgroups of index $n$ in $\pi_{1}\left(\mathcal{G}_{3}\right)$ for each isomorphism type. That is the number of non-equivalent $n$-fold covering $\mathcal{G}_{3}$, which have a prescribe fundamental group.

Theorem 2. Let $\mathcal{N} \rightarrow \mathcal{G}_{3}$ be an $n$-fold covering over $\mathcal{G}_{3}$. If $n$ is not divisible by 3 then $\mathcal{N}$ is homeomorphic to $\mathcal{G}_{3}$. If $n$ is divisible by 3 then $\mathcal{N}$ is homeomorphic to either $\mathcal{G}_{3}$ or $\mathcal{G}_{1}$. The corresponding numbers of nonequivalent coverings are given by the following formulas:

$$
\begin{aligned}
& c_{\pi_{1}\left(\mathcal{G}_{3}\right), \pi_{1}\left(\mathcal{G}_{3}\right)}(n)=\sum_{k \mid n} \theta(k)+\sum_{k \mid \frac{n}{3}} \theta(k)-2 \sum_{k \mid \frac{n}{9}} \theta(k) \\
& c_{\mathbb{Z}^{3}, \pi_{1}\left(\mathcal{G}_{3}\right)}(n)=\frac{1}{3}\left(\omega\left(\frac{n}{3}\right)+2 \sum_{k \mid \frac{n}{3}} \theta(k)+4 \sum_{k \mid \frac{n}{9}} \theta(k)\right) .
\end{aligned}
$$


The next two theorems are analogues of Theorem 1 and Theorem 2 respectively for the manifold $\mathcal{G}_{5}$.

Theorem 3. Every subgroup $\Delta$ of finite index $n$ in $\pi_{1}\left(\mathcal{G}_{5}\right)$ is isomorphic to either $\pi_{1}\left(\mathcal{G}_{5}\right)$ or $\pi_{1}\left(\mathcal{G}_{3}\right)$ or $\pi_{1}\left(\mathcal{G}_{2}\right)$ or $\pi_{1}\left(\mathcal{G}_{1}\right) \cong \mathbb{Z}^{3}$. The respective numbers of subgroups are

$$
\begin{gathered}
s_{\pi_{1}\left(\mathcal{G}_{5}\right), \pi_{1}\left(\mathcal{G}_{5}\right)}(n)=\sum_{k \mid n,\left(\frac{n}{k}, 6\right)=1} k \theta(k) \\
s_{\pi_{1}\left(\mathcal{G}_{3}\right), \pi_{1}\left(\mathcal{G}_{5}\right)}(n)=\sum_{k \mid \frac{n}{2}} k \theta(k)-\sum_{k \mid \frac{n}{6}} k \theta(k) \\
s_{\pi_{1}\left(\mathcal{G}_{2}\right), \pi_{1}\left(\mathcal{G}_{5}\right)}(n)=\omega\left(\frac{n}{3}\right)-\omega\left(\frac{n}{6}\right) \\
s_{\mathbb{Z}^{3}, \pi_{1}\left(\mathcal{G}_{5}\right)}(n)=\omega\left(\frac{n}{6}\right) .
\end{gathered}
$$

Theorem 4. The numbers of $n$-fold covering over $\mathcal{G}_{5}$ is given by the following formulas:

(iii) $\quad c_{\pi_{1}\left(\mathcal{G}_{2}\right), \pi_{1}\left(\mathcal{G}_{5}\right)}(n)=\frac{1}{3}\left(\sigma_{2}\left(\frac{n}{3}\right)+2 \sigma_{2}\left(\frac{n}{6}\right)-3 \sigma_{2}\left(\frac{n}{12}\right)+2 \sum_{k \mid \frac{n}{3}} \theta(k)-2 \sum_{k \mid \frac{n}{6}} \theta(k)\right)$

(iv). $\quad c_{\mathbb{Z}^{3}, \pi_{1}\left(\mathcal{G}_{5}\right)}(n)=\frac{1}{6}\left(\omega\left(\frac{n}{6}\right)+\sigma_{2}\left(\frac{n}{6}\right)+3 \sigma_{2}\left(\frac{n}{12}\right)+4 \sum_{k \mid \frac{n}{6}} \theta(k)+4 \sum_{k \mid \frac{n}{18}} \theta(k)\right)$

In the Appendix we present the Dirichlet generating functions for the above sequences.

\section{Preliminaries}

Further we use the representations for the fundamental groups $\pi\left(\mathcal{G}_{3}\right)$ and $\pi\left(\mathcal{G}_{5}\right)$ given in [25] and [3].

$$
\begin{gathered}
\pi_{1}\left(\mathcal{G}_{3}\right)=\left\langle x, y, z: x y x^{-1} y^{-1}=1, z x z^{-1}=y, z y z^{-1}=(x y)^{-1}\right\rangle . \\
\pi_{1}\left(\mathcal{G}_{5}\right)=\left\langle\tilde{x}, \tilde{y}, \tilde{z}: \tilde{x} \tilde{y} \tilde{x}^{-1} \tilde{y}^{-1}=1, \tilde{z} \tilde{x} \tilde{z} \tilde{z}^{-1}=\tilde{x} \tilde{y}, \tilde{z} \tilde{y} \tilde{z}^{-1}=\tilde{x}^{-1}\right\rangle .
\end{gathered}
$$

We will widely use the following statements. 
Proposition 1. Let $\Delta$ be a subgroup of finite index $n$ in $\mathbb{Z}^{2}$. Then $\Delta$ have a pair of generators of the form $(a, 0)$ and $(\mu, b)$ where $a$ and $b$ are positive integers with $a b=n$ and $\mu$ is a nonnegative integer with $0 \leq \mu<a$. Furthermore, the set of subgroups $\Delta$ with $\left|\mathbb{Z}^{2}: \Delta\right|=n$ bijectively corresponds to the set of pairs of generators of described form. The number of such subgroups $\Delta$ is $\sigma_{1}(n)$.

Let $\Delta$ be a subgroup of finite index $n$ in $\mathbb{Z}^{3}$. Then $\Delta$ have a set of three generators $(a, 0,0),(\mu, b, 0)$ and $(\nu, \lambda, c)$ where $a, b, c$ are positive integers with abc $=n, \mu$, $\nu$ are integers with $0 \leq \mu, \nu<a$ and $\lambda$ is an integer with $0 \leq \lambda<b$. Furthermore, the set of subgroups $\Delta$ with $\left|\mathbb{Z}^{3}: \Delta\right|=n$ bijectively corresponds to the set of triplets of generators of described form. The number of such subgroups $\Delta$ is $\omega(n)$.

Corollary 1. Given an integer $n$, by $S(n)$ denote the number of pairs $(H, \nu)$, where $H$ is a subgroup of index $n$ in $\mathbb{Z}^{2}$ and $\nu$ is a coset of $\mathbb{Z}^{2} / H$ with $2 \nu=0$ (we use the additive notation). Then

$$
S(n)=\sigma_{1}(n)+3 \sigma_{1}\left(\frac{n}{2}\right) .
$$

Lemma 1. Let $H \leqslant G$ be an abelian group and its subgroup of finite index. Let $\phi: G \rightarrow$ $G$ be an endomorphism of $G$, such that $\phi(H) \leqslant H$ and the index $|G: \phi(G)|$ is also finite. Then the cardinality of kernel of $\phi: G / H \rightarrow G / H$ equals to the index $|G:(H+\phi(G))|$.

For the proofs, see Lemma 1 in [2]

Remark 1. Combining Lemma 1 and Corollary 1 we get the following observation. Given a subgroup $H \leqslant \mathbb{Z}^{2}$, the number of $\nu \in \mathbb{Z}^{2} / H$, such that $2 \nu=0$, is equal to $\left|\mathbb{Z}^{2} /\langle(2,0),(0,2), H\rangle\right|$. Indeed, taking $\mathbb{Z}^{2}$ as $G, H$ as $H$ and $\phi: g \rightarrow 2 g, g \in \mathbb{Z}^{2}$ as $\phi$ one gets the desired equality. Since for each $H$ the numbers $\left|\left\{\nu \mid \nu \in \mathbb{Z}^{2} / H, 2 \nu=0\right\}\right|$ and $\left|\mathbb{Z}^{2} /\langle(2,0),(0,2), H\rangle\right|$ coincide, their sums taken over all subgroups $H$ also coincide, that is

$$
S(n)=\sum_{H \leqslant \mathbb{Z}^{2},\left|\mathbb{Z}^{2} / H\right|=n}\left|\left\{\nu \mid \nu \in \mathbb{Z}^{2} / H, 2 \nu=0\right\}\right|=\sum_{H \leqslant \mathbb{Z}^{2},\left|\mathbb{Z}^{2} / H\right|=n}\left|\mathbb{Z}^{2} /\langle(2,0),(0,2), H\rangle\right| .
$$

Definition 1. Consider the group $\mathbb{Z}^{2}$. By $\ell$ denote the automorphism $\ell: \mathbb{Z}^{2} \rightarrow \mathbb{Z}^{2}$ given by $(x, y) \rightarrow(-y, x-y)$.

Lemma 2. A subgroup $H \leqslant \mathbb{Z}^{2}$ is preserved by $\ell$ if and only if $H$ is generated by a pair of elements of the form $(p, q),(-q, p-q)$. In this case $\left|\mathbb{Z}^{2} / H\right|=p^{2}-p q+q^{2}$. For a given integer $n$ the number of invariant under $\ell$ subgroups $H$ of index $n$ in $\mathbb{Z}^{2}$ is $\theta(n)$.

Proof. Suppose $H$ is generated by elements $(p, q)$ and $(-q, p-p)=\ell((p, q))$. Then obviously $\ell(H)=H$. Also $\left|\mathbb{Z}^{2} / H\right|=p^{2}-p q+q^{2}$, since $p^{2}-p q+q^{2}$ is the number of integer points in a fundamental domain of $H$ on the plane.

Vice versa, suppose $\ell(H)=H$. Denote $d(x, y)=x^{2}-x y+y^{2}$. Let $u=(p, q) \in H \backslash\{0\}$ be an element with the minimal value of $d(u)$. Consider the subgroup $H_{1}=\langle u, \ell(u)\rangle \leqslant$ $H$. Assume $H_{1} \neq H$ and $v \in H \backslash H_{1}$. Since $H_{1}=\langle u, \ell(u)\rangle$, the fundamental domain of $H_{1}$ is a parallelogram with vertices $0, u, \ell(u), u+\ell(u)$. That means that the plane splits 
into the parallelograms of the form $w, w+u, w+\ell(u), w+u+\ell(u), w \in H_{1}$, each of them splits into two right triangles. One of this triangles contains $v$. Note that the distance from a point inside a right triangle to one of its vertices is not greater then the side of this triangle. This contradicts the minimality of $d(u)$, thus $H_{1}=H$.

To find the number of subgroups $H$ note that the number of pairs $(p, q)$ with $p^{2}-$ $p q+q^{2}=n, p>0, q \geq 0$ is $\theta(n)$. As it was proven above, for each pair $(p, q)$ of the above type two pairs $(p, q)$ and $\ell((p, q))$ generate a subgroup $H$ of the required type. Moreover $d(p, q)$ takes the minimal value among $d(v), v \in H \backslash\{0\}$. Suppose two different pairs $(p, q)$ and $\left(p^{\prime}, q^{\prime}\right)$ correspond the same subgroup $H$. Then $\left(p-p^{\prime}, q-q^{\prime}\right) \in H$ and $0<d\left(p-p^{\prime}, q-q^{\prime}\right)<d(p, q)$, which contradiction proves that there is a one-to-one correspondence between pairs $(p, q)$ and subgroups $H$.

Before formulating the next corollary note that $\ell(\nu), \nu \in \mathbb{Z}^{2} / H$ is well-defined if $\ell(H) \leq H$.

Corollary 2. Let $n$ be an integer. Consider the set of all subgroups $H$ of Given an integer $n$, by $T(n)$ denote the number of pairs $(H, \nu)$, where $H$ is a subgroup of index $n$ in $\mathbb{Z}^{2}$ with $\ell(H)=H$ and $\nu$ is a coset of $\mathbb{Z}^{2} / H$ with $\ell(\nu)=\nu$. Then

$$
T(n)=\theta(n)+2 \theta\left(\frac{n}{3}\right) .
$$

Proof. Consider subgroup $H \leqslant \mathbb{Z}^{2}$ with $\left|\mathbb{Z}^{2}: H\right|=n$ and $\ell(H)=H$. Lemma 2 claims that $H$ has a pair of generators $(p, q),(-q, p-q)$, where $p^{2}-p q+q^{2}=n$. Suppose $\ell(\nu)=\nu$ holds for some coset $\nu \in \mathbb{Z}^{2} / H$. Let $(a, b) \in \mathbb{Z}^{2}$ be a representative of coset $\nu$. Then $\nu-\ell(\nu)=(a+b,-a+2 b) \in i(p, q)+j(-q, p-q)$. That is $(a, b)=$ $i\left(\frac{2 p-q}{3}, \frac{p+q}{3}\right)+j\left(\frac{-p-q}{3}, \frac{p-2 q}{3}\right)$ for some integer $i, j$. Then modulo $\langle(p, q),(-q, p)\rangle$ there are only three different choices for pairs $(a, b)$ corresponding to $i=j=0, i=j=1$ and $i=j=2$. The first pair is always integer, the latter two are integer if and only if $p+q \equiv 0 \bmod 3$. Also, $p+q \equiv 0 \bmod 3$ if and only if $3 \mid p^{2}-p q+q^{2}=n$. That is, for a fixed $H$ there is one choice of $\nu$ if $3 \nmid n$ and three choices if $3 \mid n$. By Lemma 2 , the number of possible subgroups $H$ is $\theta(n)$. So $R(n)=\theta(n)$ if $3 \nmid n$ and $R(n)=3 \theta(n)$ if $3 \mid n$. Finally note $\theta\left(\frac{n}{3}\right)=\theta(n)$ if $3 \mid n$ and $\theta\left(\frac{n}{3}\right)=0$ otherwise. Then we have the required $R(n)=\theta(n)+2 \theta\left(\frac{n}{3}\right)$.

Remark 2. Similar to Remark 1 get

$T(n)=\sum_{\ell(H)=H<\mathbb{Z}^{2},\left|\mathbb{Z}^{2} / H\right|=n}\left|\left\{\nu \mid \nu \in \mathbb{Z}^{2} / H, \ell(\nu)=\nu\right\}\right|=\sum_{\ell(H)=H<\mathbb{Z}^{2},\left|\mathbb{Z}^{2} / H\right|=n}\left|\mathbb{Z}^{2} /\langle(1,-1),(1,2), H\rangle\right|$.

\section{On the covering of $\mathcal{G}_{3}$}

\subsection{The structure of the group $\pi_{1}\left(\mathcal{G}_{3}\right)$}

The following proposition provides the canonical form of an element in $\pi_{1}\left(\mathcal{G}_{3}\right)=\langle x, y, z$ : $\left.x y x^{-1} y^{-1}=1, z x z^{-1}=y, z y z^{-1}=(x y)^{-1}\right\rangle$. The proof is similar to the proof of Proposition 2 in [2]. 
Proposition 2. (i) Each element of $\pi_{1}\left(\mathcal{G}_{3}\right)$ can be represented in the canonical form $x^{a} y^{b} z^{c}$ for some integer $a, b, c$.

(ii) The product of two canonical forms is given by the formula

$$
x^{a} y^{b} z^{c} \cdot x^{d} y^{e} z^{f}=\left\{\begin{array}{cccc}
x^{a+d} y^{b+e} z^{c+f} & \text { if } & c \equiv 0 & \bmod 3 \\
x^{a-e} y^{b+d-e} z^{c+f} & \text { if } & c \equiv 1 & \bmod 3 \\
x^{a-d+e} y^{b-d} z^{c+f} & \text { if } & c \equiv 2 & \bmod 3
\end{array}\right.
$$

(iii) The canonical epimorphism $\phi_{\mathcal{G} 3}: \pi_{1}\left(\mathcal{G}_{3}\right) \rightarrow \pi_{1}\left(\mathcal{G}_{3}\right) /\langle x, y\rangle \cong \mathbb{Z}$, given by the formula $x^{a} y^{b} z^{c} \rightarrow c$ is well-defined.

(iv) The representation in the canonical form $g=x^{a} y^{b} z^{c}$ for each element $g \in \pi_{1}\left(\mathcal{G}_{3}\right)$ is unique.

Routinely follows from the definition of the group.

Notation. By $\Gamma$ denote the subgroup of $\pi_{1}\left(\mathcal{G}_{3}\right)$ generated by $x, y$.

Our goal is to introduce some easy invariants, similar to those of Proposition 1 .

Definition 2. Suppose all elements of $\pi_{1}\left(\mathcal{G}_{3}\right)$ are represented in the canonical form. Let $\Delta$ be a subgroup of finite index $n$ in $\pi_{1}\left(\mathcal{G}_{3}\right)$. Put $H(\Delta)=\Delta \cap \Gamma$. By $a(\Delta)$ denote the minimal positive exponent of $z$ among all the elements of $\Delta$. Choose an element $Z_{\Delta}$ with such exponent of $z$, represented in the form $Z_{\Delta}=h z^{a(\Delta)}$, where $h \in \Gamma$. By $\nu(\Delta)=h H(\Delta)$ denote the coset in the coset decomposition $\Gamma / H(\Delta)$.

Note that the invariants $a(\Delta), H(\Delta)$ and $\nu(\Delta)$ are well-defined. In particular the latter one does not depends on a choice of $Z_{\Delta}$. Also $a(\Delta)[\Gamma: H(\Delta)]=\left[\pi_{1}\left(\mathcal{G}_{2}\right): \Delta\right]$.

Definition 3. A 3-plet $(a, H, \nu)$ is called n-essential if the following conditions holds:

(i) a is a positive divisor of $n$,

(ii) $H$ is a subgroup of index $n / a$ in $\Gamma$ with $H \triangleleft \pi_{1}\left(\mathcal{G}_{3}\right)$ if $3 \nmid a$,

(iii) $\nu$ is an element of $\Gamma / H$.

The next proposition show that the introduced invariants are sufficient to enumerate the subgroups of finite index.

Proposition 3. There is a bijection between the set of n-essential 3-plets $(a, H, \nu)$ and the set of subgroups $\Delta$ of index $n$ in $\pi_{1}\left(\mathcal{G}_{5}\right)$, such that $(a, H, \nu)=(a(\Delta), H(\Delta), \nu(\Delta))$, given by the correspondence $\Delta \leftrightarrow(a(\Delta), H(\Delta), \nu(\Delta))$. Moreover, $\Delta \cong \mathbb{Z}^{3}$ if $3 \mid a(\Delta)$ and $\Delta \cong \pi_{1}\left(\mathcal{G}_{3}\right)$ otherwise.

The next few lemmas are auxiliary statements needed for the proof of Proposition 3 ,

Lemma 3. If $3 \nmid a(\Delta)$ then $H(\Delta) \triangleleft \pi_{1}\left(\mathcal{G}_{3}\right)$. 
Proof. Recall $Z_{\Delta}=h z^{a(\Delta)} \in \Delta$. Then $H(\Delta)^{Z_{\Delta}}=H(\Delta)$. Since $H(\Delta)^{x}=H(\Delta)^{y}=$ $H(\Delta)^{z^{3}}=H(\Delta)$, the former fact yields $H(\Delta)^{g}=H(\Delta), g \in \pi_{1}\left(\mathcal{G}_{3}\right)$.

Lemma 4. For arbitrary $n$-essential 3-plet $(a, H, \nu)$ there exists a subgroup $\Delta$ in the group $\pi_{1}\left(\mathcal{G}_{3}\right)$ such that $(a, H, \nu)=(a(\Delta), H(\Delta), \nu(\Delta))$.

Proof. Take an $n$-essential 3 -plet $(a, H, \nu)$. In case $3 \nmid a$ consider the following construction

$$
\Delta=\left\{h z^{(3 l+1) a(\Delta)} H \mid l \in \mathbb{Z}\right\} \bigcup\left\{h z^{a(\Delta)} h z^{-a(\Delta)} z^{(3 l+2) a(\Delta)} H \mid l \in \mathbb{Z}\right\} \bigcup\left\{z^{3 l a(\Delta)} H \mid l \in \mathbb{Z}\right\} .
$$

One can check that $\Delta$ is a subgroup of index $n$ in $\pi_{1}\left(\mathcal{G}_{3}\right)$ and $(a(\Delta), H(\Delta), \nu(\Delta))=$ $(a, H, \nu)$.

Similarly, in case $3 \mid a$ the set

$$
\Delta=\left\{h^{l} z^{l a(\Delta)} H \mid l \in \mathbb{Z}\right\}
$$

is the required subgroup.

Proof of Proposition [3. Consider the family of subgroups $\Delta$ of index $n$ in $\pi_{1}\left(\mathcal{G}_{3}\right)$, and the family of $n$-essential 3-plets. The definition of notions $a(\Delta), H(\Delta)$ and $\nu(\Delta)$ together with Lemma 3 provide the correspondence from subgroups to $n$-essential 3 -plets. Since the above invariants are well-defined, each subgroup $\Delta$ corresponds to only one 3-plet. By virtue of Lemma 4 each 3-plet corresponds to some subgroup, also different 3-plets correspond to different 3-plets. The bijection part is proven.

If $3 \mid a(\Delta)$ Lemma 4 implies that $\Delta$ is a subgroup of $\left\langle x, y, z^{3}\right\rangle$. Thus $\Delta$ is a subgroup of finite index in $\mathbb{Z}^{3}$, hence $\Delta$ is isomorphic to $\mathbb{Z}^{3}$ itself.

Consider case $a(\Delta) \equiv 1 \bmod 3$. Since $H(\Delta)$ is a subgroup of finite index in $\langle x, y\rangle \cong$ $\mathbb{Z}^{2}$, we have $H(\Delta) \cong \mathbb{Z}^{2}$. By Lemmas 2 and 3 there is a pair of elements, that generates $H(\Delta)$, that have the form form $\left(x^{p} y^{q}, x^{-q} y^{p-q}\right)$. Let $h$ be an arbitrary element in the coset $\nu(\Delta)$. Put $X=x^{p} y^{q}, Y=x^{-q} y^{p-q}$ and $Z=h z^{a(\Delta)}$. Direct verification shows that the relations $X Y X^{-1} Y^{-1}=1, Z X Z^{-1}=Y$, and $Z Y Z^{-1}=(X Y)^{-1}$ holds. Further we call this relations the proper relations of the subgroup $\Delta$. Thus the map $x \rightarrow X, y \rightarrow Y, z \rightarrow$ $Z$ can be extended to an epimorphism $\pi_{1}\left(\mathcal{G}_{3}\right) \rightarrow \Delta$. To prove that this epimorphism is really an isomorphism we need to show that each relation in $\Delta$ is a corollary of proper relations. We call a relation, that is not a corollary of proper relations an improper relation.

Assume the contrary, i.e. there are some improper relations in $\Delta$. Since in $\Delta$ the proper relations holds, each element can be represented in the canonical form, given by Proposition 2 in terms of $X, Y, Z$, by using just the proper relations. That is each element $g$ can be represented as

$$
g=X^{a} Y^{b} Z^{c} .
$$

If there is an improper relation then there is an equality

$$
X^{a} Y^{b} Z^{c}=X^{a^{\prime}} Y^{b^{\prime}} Z^{c^{\prime}},
$$


where at least one of the inequalities $a \neq a^{\prime}, b \neq b^{\prime}, c \neq c^{\prime}$ holds. Applying $\phi_{\mathcal{G} 3}$ to both parts we get $c a(\Delta)=c^{\prime} a(\Delta)$, thus $c=c^{\prime}$. Then $U^{a} V^{b}=U^{a^{\prime}} V^{b^{\prime}}$, that means

$$
\left\{\begin{array}{r}
a p-b q=a^{\prime} p-b^{\prime} q \\
a q+b(p-q)=a^{\prime} q+b^{\prime}(p-q)
\end{array}\right.
$$

Since $\operatorname{det}\left(\begin{array}{cc}p & -q \\ q & p-q\end{array}\right)=p^{2}-p q+q^{2}=\frac{n}{a(\Delta)} \neq 0$, the system 3.5 implies $a=a^{\prime}$ and $b=b^{\prime}$, this contradiction proves that $\pi_{1}\left(\mathcal{G}_{3}\right) \cong \Delta$.

The proof in case $a(\Delta) \equiv 2 \bmod 3$ is similar with the only difference we take $Y=$ $x^{p} y^{q}$ and $X=x^{-q} y^{p-q}$.

\subsection{The proof of Theorem 1}

Proceed to the proof of Theorem 1. Proposition 3 claims that each subgroup $\Delta$ of finite index $n$ is isomorphic to $\pi_{1}\left(\mathcal{G}_{3}\right)$ or $\mathbb{Z}^{3}$, depending upon whether $a(\Delta)$ is a multiple of 3 . Consider these two cases separately.

Case (i). Let $\Delta$ be a subgroup of $\pi_{1}\left(\mathcal{G}_{3}\right)$ isomorphic to $\pi_{1}\left(\mathcal{G}_{3}\right)$. To find the number of such subgroups, by Proposition 3 we need to calculate the cardinality of the set of $n$-essential 3-plets with $3 \nmid a$.

For each $3 \nmid a$ there are $\theta\left(\frac{n}{a}\right)$ subgroups $H$ in $\Gamma$ such that $|\Gamma: H|=\frac{n}{a}$ and $H \triangleleft \pi_{1}\left(\mathcal{G}_{3}\right)$. Also there are $\frac{n}{a}$ different choices of a coset $\nu$. Thus, for each $3 \nmid a$ the number of $n$ essential 3-plets is $\frac{n}{a} \theta\left(\frac{n}{a}\right)$. So, the total number of subgroups is given by

$$
s_{\pi_{1}\left(\mathcal{G}_{3}\right), \pi_{1}\left(\mathcal{G}_{3}\right)}(n)=\sum_{a \mid n, 3 \nmid a} \frac{n}{a} \theta\left(\frac{n}{a}\right)=\sum_{a \mid n} \frac{n}{a} \theta\left(\frac{n}{a}\right)-\sum_{3 a \mid n} \frac{n}{3 a} \theta\left(\frac{n}{3 a}\right)=\sum_{k \mid n} k \theta(k)-\sum_{k \mid \frac{n}{3}} k \theta(k) .
$$

Case (ii). Similarly to the previous case, we get the formula

$$
s_{\mathbb{Z}^{3}, \pi_{1}\left(\mathcal{G}_{3}\right)}(n)=\sum_{3 a \mid n} \frac{n}{3 a} \sigma_{1}\left(\frac{n}{3 a}\right)=\omega\left(\frac{n}{3}\right) .
$$

\subsection{The proof of Theorem 2}

\subsubsection{Overall scheme of the proof}

The proof of both cases follows the general scheme that we describe first. Recall that a subgroup $G$ of finite index in $\pi_{1}\left(\mathcal{G}_{3}\right)$ has one of the following isomorphism types: $\mathbb{Z}^{3}$ or $\pi_{1}\left(\mathcal{G}_{3}\right)$. We use the standard notation $\left[g_{1}, g_{2}\right]=g_{1} g_{2} g_{1}^{-1} g_{2}^{-1}, g_{1}, g_{2} \in \pi_{1}\left(\mathcal{G}_{3}\right)$. Also, given subgroups $G_{1}, G_{2} \leqslant \pi_{1}\left(\mathcal{G}_{3}\right)$ by $\left[G_{1}, G_{2}\right]$ we denote the subgroup, generated by the elements $\left[g_{1}, g_{2}\right], g_{1} \in G_{1}, g_{2} \in G_{2}$. Fix an isomorphism type of a subgroup. Further $\Delta$ will always denote a subgroup of this isomorphism type of index $n$ in $\pi_{1}\left(\mathcal{G}_{3}\right)$. In each case we point a normal subgroup of finite index $\Lambda \unlhd \pi_{1}\left(\mathcal{G}_{3}\right)$ such that two conditions are met: 
$1^{\circ}$ for any $\lambda \in \Lambda$ and any $\Delta$ holds $A d_{\lambda}(H(\Delta))=H(\Delta)$,

$2^{\circ}[\Lambda, Z(\Delta)]=[H(\Lambda), Z(\Delta)]$, where $Z(\Delta)$ is given by Definition 2 ,

Call an intermediate conjugacy class $\Delta^{\Lambda}$ of $\Delta$ the set of subgroups $\Delta^{\lambda}, \lambda \in \Lambda$. Denote the number of such classes by $c_{G, \pi_{1}\left(\mathcal{G}_{3}\right)}^{\Lambda}$, where $G$ isomorphic to one of $\mathbb{Z}^{3}$ or $\pi_{1}\left(\mathcal{G}_{3}\right)$.

We propose an algorithm to uniformly calculate $c_{G, \pi_{1}\left(\mathcal{G}_{3}\right)}^{\Lambda}$. Given $\Delta$, a subgroup $\Delta^{\prime} \in \Delta^{\Lambda}$ have the following invariants: $a\left(\Delta^{\prime}\right)=a(\Delta), H\left(\Delta^{\prime}\right)=H(\Delta)$ and $\nu\left(\Delta^{\prime}\right) \in$ $\nu(\Delta)[\Lambda, Z(\Delta)]$. Keep in mind that $[\Lambda, Z(\Delta)] \leqslant \Gamma$, since $\Gamma$ is normal in $\pi_{1}\left(\mathcal{G}_{3}\right)$ and $\pi_{1}\left(\mathcal{G}_{3}\right) / \Gamma$ is abelian. Thus for a fixed pair $(a, H)$ there are $|\Gamma:\langle[\Lambda, Z(\Delta)], H\rangle|$ partial conjugacy classes $\Delta^{\Lambda}$, each corresponding to the pair $(a, H)$. This let us to enumerate partial conjugacy classes.

The factor-group $\pi_{1}\left(\mathcal{G}_{3}\right) / \Lambda$ acts by conjugation on partial conjugacy classes. An orbit of partial conjugacy classes form a conjugacy class, thus we use the Burnside's lemma to calculate the number of conjugacy classes. To do this, we introduce one more definition.

Definition 4. Given $u \in \pi_{1}\left(\mathcal{G}_{3}\right) / \Lambda$, by $B(u)$ denote the number of partial conjugacy classes, preserved by the conjugation with $u: B(u)=\left|\left\{\Delta^{\Lambda} \mid\left(\Delta^{\Lambda}\right)^{u}=\Delta^{\Lambda}\right\}\right|$. In particular, $B(1)$ is the number of partial conjugacy classes.

Now we are done with the general scheme and proceed to its realization in specific cases.

\subsubsection{Case (i)}

Put $\Lambda=\pi_{1}\left(\mathcal{G}_{3}\right)$. Proposition 3 claims $H(\Delta) \triangleleft \pi_{1}\left(\mathcal{G}_{3}\right)$ in case $\Delta \cong \pi_{1}\left(\mathcal{G}_{3}\right)$, thus $\left(1^{\circ}\right)$ holds. Recall that $Z(\Delta)=h z^{a(\Delta)}$. Direct calculation through (3.3) shows that $[\Lambda, Z(\Delta)]=\left\langle x y^{-1}, x y^{2}\right\rangle=[H(\Lambda), Z(\Delta)]$.

This means, firstly, that $\left(2^{\circ}\right)$ holds, and secondly that $|\Gamma:\langle[\Lambda, Z(\Delta)], H\rangle|$ equals 1 if $3 \nmid n$ and equals 3 if $3 \mid n$. For a fixed $a(\Delta)$ the number of subgroups $H(\Delta)$ is $\theta\left(\frac{n}{a(\Delta)}\right)$. Keep in mind that $\theta\left(\frac{k}{3}\right)=\theta(k)$ if $\frac{k}{3}$ is integer, and $\theta\left(\frac{k}{3}\right)=0$ otherwise. So the function $k \mapsto\left\{\begin{aligned} \theta(k) & \text { if } 3 \nmid k \\ 3 \theta(k) & \text { if } 3 \mid k\end{aligned}\right.$ is given by $\theta(k)+2 \theta \frac{k}{3}$. Applying this and summing achieved number of pairs $(H, \nu)$ over all values of $a$ one gets:

$$
\begin{aligned}
& c_{\pi_{1}\left(\mathcal{G}_{3}\right), \pi_{1}\left(\mathcal{G}_{3}\right)}(n)=\sum_{a \mid n, 3 \nmid a} \theta\left(\frac{n}{a}\right)+2 \theta\left(\frac{n}{3 a}\right)=\sum_{a \mid n} \theta\left(\frac{n}{a}\right)+\sum_{a \mid \frac{n}{3}} \theta\left(\frac{n}{3 a}\right)-2 \sum_{a \mid \frac{n}{9}} \theta\left(\frac{n}{9 a}\right)= \\
& \sum_{k \mid n} \theta(k)+\sum_{k \mid \frac{n}{3}} \theta(k)-2 \sum_{k \mid \frac{n}{9}} \theta(k) .
\end{aligned}
$$

Since $\Lambda=\pi_{1}\left(\mathcal{G}_{3}\right)$, the Burnside's lemma is not needed to conclude $c_{\pi_{1}\left(\mathcal{G}_{3}\right), \pi_{1}\left(\mathcal{G}_{3}\right)}(n)=$ $c_{\pi_{1}\left(\mathcal{G}_{3}\right), \pi_{1}\left(\mathcal{G}_{3}\right)}^{\Lambda}(n)$. 


\subsubsection{Case (ii)}

Put $\Lambda=\left\langle x, y, z^{3}\right\rangle$. Since $\Lambda \cong \mathbb{Z}^{3}$ and $\Delta \leqslant \Lambda$ for any $\Delta$, conditions $\left(1^{\circ}\right)$ and $\left(2^{\circ}\right)$ hold. Also each partial conjugacy class consists of one subgroup, i.e. $c_{\mathbb{Z}^{3}, \pi_{1}\left(\mathcal{G}_{3}\right)}^{\Lambda}(n)=$ $s_{\mathbb{Z}^{3}, \pi_{1}\left(\mathcal{G}_{3}\right)}(n)=\omega\left(\frac{n}{3}\right)$.

The factor $\pi_{1}\left(\mathcal{G}_{3}\right) / \Lambda$ consists of three elements, represented by $1, z$, and $z^{2}$ respectively.

The condition $\Delta^{z}=\Delta$ is equivalent to $(H(\Delta))^{z}=H(\Delta)$ and $(\nu(\Delta))^{z}=\nu(\Delta)$ met simultaneously. Corollary 2 provides the number of such pairs $(H, \nu)$ for a given value of $a$. Summing over all the possible values (recall that $3 \mid a$ ) one gets $B(z)=B\left(z^{2}\right)=$ $\sum_{k \mid \frac{n}{3}} \theta(k)+2 \sum_{k \mid \frac{n}{9}} \theta(k)$.

By making use of Burnside's lemma obtain

$$
c_{\mathbb{Z}^{3}, \pi_{1}\left(\mathcal{G}_{3}\right)}(n)=\frac{1}{3}\left(\omega\left(\frac{n}{3}\right)+2 \sum_{k \mid \frac{n}{3}} \theta(k)+4 \sum_{k \mid \frac{n}{9}} \theta(k)\right) .
$$

\section{On the coverings of $\mathcal{G}_{5}$}

\subsection{The structure of the group $\pi_{1}\left(\mathcal{G}_{5}\right)$}

Recall that the fundamental group of $\mathcal{G}_{5}$ is given by: $\pi_{1}\left(\mathcal{G}_{5}\right)=\left\langle\tilde{x}, \tilde{y}, \tilde{z}: \tilde{x} \tilde{y} \tilde{x}^{-1} \tilde{y}^{-1}=\right.$ $\left.1, \tilde{z} \tilde{x} \tilde{z}^{-1}=\tilde{x} \tilde{y}, \tilde{z} \tilde{y} \tilde{z}^{-1}=\tilde{x}^{-1}\right\rangle$. The following proposition provides the canonical form of an element in $\pi_{1}\left(\mathcal{G}_{5}\right)$.

Proposition 4. (i) Each element of $\pi_{1}\left(\mathcal{G}_{5}\right)$ can be represented in the canonical form $\tilde{x}^{a} \tilde{y}^{b} \tilde{z}^{c}$ for some integer $a, b, c$.

(ii) The product of two canonical forms is given by the formula

$$
\tilde{x}^{a} \tilde{y}^{b} \tilde{z}^{c} \cdot \tilde{x}^{d} \tilde{y}^{e} \tilde{z}^{f}=\left\{\begin{array}{cccc}
\tilde{x}^{a+d} \tilde{y}^{b+e} \tilde{z}^{c+f} & \text { if } & c \equiv 0 & \bmod 6 \\
\tilde{x}^{a+d-e} \tilde{y}^{b+d} \tilde{z}^{c+f} & \text { if } & c \equiv 1 & \bmod 6 \\
\tilde{x}^{a-e} \tilde{y}^{b+d-e} \tilde{z}^{c+f} & \text { if } & c \equiv 2 & \bmod 6 \\
\tilde{x}^{a-d} \tilde{y}^{b-e} \tilde{z}^{c+f} & \text { if } & c \equiv 3 & \bmod 6 \\
\tilde{x}^{a-d+e} \tilde{y}^{b-d} \tilde{z}^{c+f} & \text { if } & c \equiv 4 & \bmod 6 \\
\tilde{x}^{a+e} \tilde{y}^{b-d+e} \tilde{z}^{c+f} & \text { if } & c \equiv 5 & \bmod 6
\end{array}\right.
$$

(iii) The canonical epimorphism $\phi_{\mathcal{G}_{5}}: \pi_{1}\left(\mathcal{G}_{5}\right) \rightarrow \pi_{1}\left(\mathcal{G}_{5}\right) /\langle\tilde{x}, \tilde{y}\rangle \cong \mathbb{Z}$, given by the formula $\tilde{x}^{a} \tilde{y}^{b} \tilde{z}^{c} \rightarrow c$ is well-defined.

(iv) The representation in the canonical form $g=\tilde{x}^{a} \tilde{y}^{b} \tilde{z}^{c}$ for each element $g \in \pi_{1}\left(\mathcal{G}_{5}\right)$ is unique.

Routinely follows from the definition of the group.

Notation. Let $\Gamma=\langle\tilde{x}, \tilde{y}\rangle$ be the subgroup of $\pi_{1}\left(\mathcal{G}_{5}\right)$ generated by $\tilde{x}, \tilde{y}$. 
Our goal is to introduce some easy invariants, similar to those in Proposition 1. That will let us to enumerate the subgroups.

Definition 5. Suppose all elements of $\pi_{1}\left(\mathcal{G}_{5}\right)$ are represented in the canonical form. Let $\Delta$ be a subgroup of finite index $n$ in $\pi_{1}\left(\mathcal{G}_{5}\right)$. Put $H(\Delta)=\Delta \bigcap \Gamma$. By $a(\Delta)$ denote the minimal positive exponent of $\tilde{z}$ among all the elements $\tilde{x}^{a} \tilde{y}^{b} \tilde{z}^{c} \in \Delta$. Choose an element $Z(\Delta)$ with such exponent of $\tilde{z}$, represented in the form $Z(\Delta)=h \tilde{z}^{a(\Delta)}$, where $h \in \Gamma$. By $\nu(\Delta)=h H(\Delta)$ denote the coset in $\Gamma / H(\Delta)$ containing $h$.

Definition 6. A 3-plet $(a, H, \nu)$ is called n-essential if the following conditions holds:

(i) a is a positive divisor of $n$,

(ii) $H$ is a subgroup of index $n / a$ in $\Gamma$ with $H \triangleleft \pi_{1}\left(\mathcal{G}_{5}\right)$ if $3 \nmid a$,

(iii) $\nu$ is an element of $\Gamma / H$.

Proposition 5. There is a bijection between the set of $n$-essential 3-plets $(a, H, \nu)$ and the set of subgroups $\Delta$ of index $n$ in $\pi_{1}\left(\mathcal{G}_{5}\right)$, given by the correspondence $\Delta \leftrightarrow(a, H, \nu)=$ $(a(\Delta), H(\Delta), \nu(\Delta))$. Moreover, $\Delta \cong \mathbb{Z}^{3}$ if $(a, 6)=6, \Delta \cong \pi_{1}\left(\mathcal{G}_{2}\right)$ if $(a, 6)=3, \Delta \cong$ $\pi_{1}\left(\mathcal{G}_{3}\right)$ if $(a, 6)=2$ and $\Delta \cong \pi_{1}\left(\mathcal{G}_{5}\right)$ if $(a, 6)=1$.

Proof. The proof of Proposition 5 is similar to the proof of Proposition 3 ,

\subsection{The proof of Theorem 3}

In case (i) the argument similar to the proof of Theorem 1 leads to the formula:

$$
s_{\pi_{1}\left(\mathcal{G}_{5}\right), \pi_{1}\left(\mathcal{G}_{5}\right)}(n)=\sum_{a \mid n,(a, 6)=1} \frac{n}{a} \theta\left(\frac{n}{a}\right)=\sum_{k \mid n,\left(\frac{n}{k}, 6\right)=1} k \theta(k) .
$$

The last equality is obtained by applying the inclusion-exclusion principle. The cases (ii), (iii) and (iv) can be treated in the similar way.

\subsection{The proof of Theorem 4}

The proof uses the overall scheme form section 3.3.1, so we just proceed to to its realization in specific cases.

\subsubsection{Case (i)}

Put $\Lambda=\pi_{1}\left(\mathcal{G}_{5}\right)$. Proposition 5 claims $H(\Delta) \triangleleft \pi_{1}\left(\mathcal{G}_{5}\right)$ in case $\Delta \cong \pi_{1}\left(\mathcal{G}_{5}\right)$, thus $\left(1^{\circ}\right)$ holds. Direct calculation using (4.6) in case $a(\Delta) \equiv 1 \bmod 6 \operatorname{gives}[\tilde{x}, Z(\Delta)]=\tilde{y}^{-1}$ and $[\tilde{y}, Z(\Delta)]=\tilde{x} \tilde{y}$. In case $c(\Delta) \equiv 5 \bmod 6$ we respectively get $[\tilde{x}, Z(\Delta)]=\tilde{x} \tilde{y}$ and $[\tilde{y}, Z(\Delta)]=\tilde{x}^{-1}$. So in both cases $[\Gamma, Z(\Delta)]=\Gamma$. Firstly this means that $\left(2^{\circ}\right)$ holds. Secondly, conjugacy classes of subgroups $\Delta$ are in one-to-one correspondence with pairs $(a, H)$. Summing the number of choices of $H$ over all the possible values of $a$ get

$$
c_{\pi_{1}\left(\mathcal{G}_{5}\right), \pi_{1}\left(\mathcal{G}_{5}\right)}(n)=\sum_{a \mid n,(6, a)=1} \theta\left(\frac{n}{a}\right)=\sum_{k \mid n} \theta(k)-\sum_{k \mid \frac{n}{2}} \theta(k)-\sum_{k \mid \frac{n}{3}} \theta(k)+\sum_{k \mid \frac{n}{6}} \theta(k) .
$$




\subsubsection{Case (ii)}

Put $\Lambda=\left\langle\tilde{x}, \tilde{y}, \tilde{z}^{2}\right\rangle$. Proposition 5 claims $H(\Delta) \triangleleft \pi_{1}\left(\mathcal{G}_{5}\right)$ in case $\Delta \cong \pi_{1}\left(\mathcal{G}_{5}\right)$, thus $\left(1^{\circ}\right)$ holds. Recall that $Z(\Delta)=h \tilde{z}^{a(\Delta)}$. Direct calculation through (4.6) shows that $[\Lambda, Z(\Delta)]=\left\langle\tilde{x} \tilde{y}^{-1}, \tilde{x} \tilde{y}^{2}\right\rangle=[H(\Lambda), Z(\Delta)]$.

This means, firstly, that $\left(2^{\circ}\right)$ holds, and secondly that $|\Gamma:\langle[\Lambda, Z(\Delta)], H\rangle|$ equals 1 if $3 \nmid n$ and equals 3 if $3 \mid n$.

The factor $\pi_{1}\left(\mathcal{G}_{5}\right) / \Lambda$ consists of two elements, represented by 1 and $\tilde{x}^{3}$ respectively. The conjugation with these elements preserves $(a, H)$, thus in case $3 \nmid n$ the partial conjugacy classes coincide with conjugacy classes, and there is only one conjugacy class for a fixed pair $(a, H)$. In case $3 \mid n$ for a fixed pair $(a, H)$ there are 3 partial conjugacy classes: namely $\Delta_{0}^{\Lambda}, \Delta_{1}^{\Lambda}$ and $\Delta_{2}^{\Lambda}$, where $\Delta_{0} \leftrightarrow(a, H, 1), \Delta_{1} \leftrightarrow(a, H, \tilde{y})$ and $\Delta_{2} \leftrightarrow$ $\left(a, H, \tilde{y}^{2}\right)$. Note that the conjugation with $\tilde{x}^{3}$ swaps the partial conjugacy classes $\Delta_{1}^{\Lambda}$ and $\Delta_{2}^{\Lambda}$. Thus for a fixed pair $(a, H)$ there are two conjugacy classes: $\Delta_{0}^{\pi_{1}\left(\mathcal{G}_{5}\right)}=\Delta_{0}^{\Lambda}$ and $\Delta_{1}^{\pi_{1}\left(\mathcal{G}_{5}\right)}=\Delta_{1}^{\Lambda} \bigcup \Delta_{2}^{\Lambda}$.

Keep in mind that $\theta\left(\frac{n}{3}\right)=\theta(n)$ if $\frac{n}{3}$ is integer, and $\theta\left(\frac{n}{3}\right)=0$ otherwise. Applying this and summing achieved number of conjugacy classes over all values of $a$ one gets:

$$
\begin{aligned}
& c_{\pi_{1}\left(\mathcal{G}_{3}\right), \pi_{1}\left(\mathcal{G}_{5}\right)}(n)=\sum_{a \mid \frac{n}{2}, a \nmid \frac{n}{6}, 3 \nmid a} \theta\left(\frac{n}{2 a}\right)+2 \sum_{a \mid \frac{n}{6}, 3 \nmid a} \theta\left(\frac{n}{6 a}\right)=\sum_{a \mid \frac{n}{2}, 3 \nmid a} \theta\left(\frac{n}{2 a}\right)+\sum_{a \mid \frac{n}{6}, 3 \nmid a} \theta\left(\frac{n}{6 a}\right)= \\
& \sum_{a \mid \frac{n}{2}} \theta\left(\frac{n}{2}\right)-\sum_{a \mid \frac{n}{18}} \theta\left(\frac{n}{18}\right)=\sum_{k \mid \frac{n}{2}} \theta(k)-\sum_{k \mid \frac{n}{18}} \theta(k) .
\end{aligned}
$$

\subsubsection{Case (iii)}

Put $\Lambda=\left\langle\tilde{x}, \tilde{y}, \tilde{z}^{3}\right\rangle . A d_{\tilde{x}}$ and $A d_{\tilde{y}}$ are the identity transformation on $\Gamma . A d_{\tilde{z}^{3}}$ is given by $g \rightarrow g^{-1}, g \in \Gamma$. That is $A d_{\tilde{x}}, A d_{\tilde{y}}$ and $A d_{\tilde{z}^{3}}$ preserves $H(\Delta)$, i.e. $\left(1^{\circ}\right)$ holds.

Further $[H(\Lambda), Z(\Delta)]=\left\langle\tilde{x}^{2}, \tilde{y}^{2}\right\rangle$. Recall the notation $Z(\Delta)=h \tilde{z}^{a(\Delta)}$. Then $\left[\tilde{z}^{3}, Z(\Delta)\right]=$ $h^{-2} \in\left\langle\tilde{x}^{2}, \tilde{y}^{2}\right\rangle$, so $\left(2^{\circ}\right)$ holds.

Applying Remark 1 and Corollary 1 and summing over all possible values of $a$ find

$$
c_{\pi_{1}\left(\mathcal{G}_{2}\right), \pi_{1}\left(\mathcal{G}_{5}\right)}^{\Lambda}(n)=\sum_{\substack{a|n, 3| a, \zeta \nmid a}}\left(\sigma_{1}\left(\frac{n}{a}\right)+3 \sigma_{1}\left(\frac{n}{2 a}\right)\right)=\sigma_{2}\left(\frac{n}{3}\right)+2 \sigma_{2}\left(\frac{n}{6}\right)-3 \sigma_{2}\left(\frac{n}{12}\right) .
$$

The factor $\pi_{1}\left(\mathcal{G}_{5}\right) / \Lambda$ consists of three elements, represented by $1, \tilde{x}^{2}$ and $\tilde{x}^{4}$ respectively. Obviously the numbers of partial conjugacy classes, preserved by $A d_{\tilde{x}^{2}}$ and $A d_{\tilde{x}^{4}}$ coincide: $B\left(\tilde{x}^{2}\right)=B\left(\tilde{x}^{4}\right)$. To find them note the following. A partial conjugacy class $\Delta^{\Lambda}$ is preserved by $A d_{\tilde{x}^{2}}$ iff the hollowing conditions are met simultaneously:

$(*)(H(\Delta))^{\tilde{z}^{2}}=H(\Delta)$

$(* *)(\nu(\Delta))^{\tilde{z}^{2}}$ must belong to the same conjugacy class in $\Gamma /\left\langle\tilde{x}^{2}, \tilde{y}^{2}, H\right\rangle$ as $\nu(\Delta)$. 
By Lemma2 the condition $(*)$ implies that $H(\Delta)$ have a pair of generators $\left(\tilde{x}^{p} \tilde{y}^{q}, \tilde{x}^{-q} \tilde{y}^{p-q}\right)$. The matrix $\left(\begin{array}{cc}p & q \\ -q & p-q\end{array}\right)$ modulo 2 have the rank 0 or 2 , never 1 . So $\Gamma /\left\langle\tilde{x}^{2}, \tilde{y}^{2}, \tilde{x}^{p} \tilde{y}^{q}, \tilde{x}^{-q} \tilde{y}^{p-q}\right\rangle$ is either trivial or isomorphic to $\mathbb{Z}_{2}^{2}$. In the first case the condition (**) holds for the sole element, in the second case the condition $(* *)$ holds only for the coset of 0 , and does not holds for three other cosets.

So the conjugacy classes $\Delta^{\Lambda}$ with $\Delta^{\tilde{z}^{2}} \in \Delta^{\Lambda}$ are in one-to-one correspondence with the normal subgroups $H(\Delta) \triangleleft \pi_{1}\left(\mathcal{G}_{5}\right)$. Applying Lemma 2 and summing over all the possible values of a yields $B\left(\tilde{x}^{2}\right)=B\left(\tilde{x}^{4}\right)=\sum_{k \mid \frac{n}{3}} \theta(k)-\sum_{k \mid \frac{n}{6}} \theta(k)$. Substituting to Burnside's lemma obtain

$$
c_{\pi_{1}\left(\mathcal{G}_{2}\right), \pi_{1}\left(\mathcal{G}_{5}\right)}(n)=\frac{1}{3}\left(\sigma_{2}\left(\frac{n}{3}\right)+2 \sigma_{2}\left(\frac{n}{6}\right)-3 \sigma_{2}\left(\frac{n}{12}\right)+2 \sum_{k \mid \frac{n}{3}} \theta(k)-2 \sum_{k \mid \frac{n}{6}} \theta(k)\right) .
$$

\subsubsection{Case (iv)}

Put $\Lambda=\left\langle\tilde{x}, \tilde{y}, \tilde{z}^{6}\right\rangle$. Since $\Lambda \cong \mathbb{Z}^{3}$ and $\Delta \leqslant \Lambda$ for any $\Delta$, conditions $\left(1^{\circ}\right)$ and $\left(2^{\circ}\right)$ hold. Also each partial conjugacy class consists of one subgroup, i.e. $c_{\mathbb{Z}^{3}, \pi_{1}\left(\mathcal{G}_{5}\right)}(n)^{\lambda}=$ $s_{\mathbb{Z}^{3}, \pi_{1}\left(\mathcal{G}_{5}\right)}(n)=\omega\left(\frac{n}{6}\right)$.

The factor $\pi_{1}\left(\mathcal{G}_{5}\right) / \Lambda$ consists of six elements, represented by $1, \tilde{x}, \tilde{x}^{2}, \tilde{x}^{3}, \tilde{x}^{4}$ and $\tilde{x}^{5}$ respectively.

The condition $\Delta^{\tilde{z}^{3}}=\Delta$ is equivalent to $2 \nu(\Delta)=0$. Corollary 1 provides the number of such pairs $(H, \nu)$ for a given value of $a$, summing over all possible values (recall that $6 \mid a)$ one gets $B\left(\tilde{z}^{3}\right)=\sigma_{2}\left(\frac{n}{6}\right)+3 \sigma_{2}\left(\frac{n}{12}\right)$.

The condition $\Delta^{\tilde{z}^{2}}=\Delta$ is equivalent to $(H(\Delta))^{\tilde{z}^{2}}=H(\Delta)$ and $(\nu(\Delta))^{\tilde{z}^{2}}=\nu(\Delta)$ met simultaneously. Corollary 1 provides the number of such pairs $(H, \nu)$ for a given value of $a$. Summing over all the possible values (recall that $6 \mid a$ ) one gets $B\left(\tilde{z}^{2}\right)=B\left(\tilde{z}^{4}\right)=$ $\sum_{k \mid \frac{n}{6}} \theta(k)+2 \sum_{k \mid \frac{n}{18}} \theta(k)$.

Finally, $\Delta^{\tilde{z}}=\Delta$ implies $(H(\Delta))^{\tilde{z}}=H(\Delta)$ and $(\nu(\Delta))^{\tilde{z}^{3}}=(\nu(\Delta))^{\tilde{z}^{2}}=\nu(\Delta)$. The latter two equalities imply $\nu(\Delta)=0$, so the unique subgroup $\Delta$ correspond to a $H(\Delta)$. Summing over all the possible values of $a$ yields $B(\tilde{z})=B\left(\tilde{z}^{5}\right)=\sum_{k \mid \frac{n}{6}} \theta(k)$.

Substituting to Burnside's lemma obtain

$$
c_{\mathbb{Z}^{3}, \pi_{1}\left(\mathcal{G}_{5}\right)}(n)=\frac{1}{6}\left(\omega\left(\frac{n}{6}\right)+\sigma_{2}\left(\frac{n}{6}\right)+3 \sigma_{2}\left(\frac{n}{12}\right)+4 \sum_{k \mid \frac{n}{6}} \theta(k)+4 \sum_{k \mid \frac{n}{18}} \theta(k)\right) .
$$

\section{Appendix}

Given a sequence $\{f(n)\}_{n=1}^{\infty}$, a formal power series

$$
\widehat{f}(s)=\sum_{n=1}^{\infty} \frac{f(n)}{n^{s}}
$$


is called Dirichlet generating function for $\{f(n)\}_{n=1}^{\infty}$, see for example, 23. For the way to reconstruct the sequence $f(n)$ by $\widehat{f}(s)$ see Perron's formula (for example [24]).

Here we present the Dirichlet generating functions for the calculated sequences $s_{H, G}(n)$ and $c_{H, G}(n)$. Since theorems 1-4 provides he explicit formulas, the remaining can is done by direct calculations which we omit here.

Notations. By $\zeta(s)$ we denote the Riemann zeta function. Define sequence $\{\chi(n)\}_{n=1}^{\infty}$ by $\chi(n)=\frac{1}{\sqrt{3}}\left(\left(-\frac{1}{2}+\frac{\sqrt{3}}{2} i\right)^{n}-\left(-\frac{1}{2}-\frac{\sqrt{3}}{2} i\right)^{n}\right)$ or equivalently $\chi(n)=\left\{\begin{array}{r}1 \text { if } n \equiv 1 \bmod 3 \\ -1 \text { if } n \equiv 2 \bmod 3\end{array}\right.$. For the sake of brevity denote $\vartheta(s)=\widehat{\chi}(s)$. Note that $\vartheta(s)$ is the Dirichlet L-series for the multiplicative character $\chi(n)$.

\begin{tabular}{|l|l|l|l|}
\hline \multicolumn{2}{|c|}{$\mathrm{G}$} & \multicolumn{1}{l|}{$\mathcal{G}_{3}$} & \multicolumn{1}{l|}{$\mathcal{G}_{5}$} \\
\hline \multirow{2}{*}{$\mathbb{Z}^{3}$} & $\widehat{s}_{H, G}$ & $3^{-s} \zeta(s) \zeta(s-1) \zeta(s-2)$ & $6^{-s} \zeta(s) \zeta(s-1) \zeta(s-2)$ \\
\cline { 2 - 5 } & $\widehat{c}_{H, G}$ & $3^{-s-1} \zeta(s)(\zeta(s-1) \zeta(s-2)+$ & $6^{-s-1} \zeta(s)(\zeta(s \quad-1) \zeta(s-2)+$ \\
& & $\left.2\left(1+2 \cdot 3^{-s}\right) \zeta(s) \vartheta(s)\right)$ & $\left.\left(1+3 \cdot 2^{-s}\right) \zeta(s) \zeta(s-1)+4\left(1+3^{-s}\right) \zeta(s) \vartheta(s)\right)$ \\
\hline \multirow{2}{*}{$\mathcal{G}_{2}$} & $\widehat{s}_{H, G}$ & & $3^{-s}\left(1-2^{-s}\right) \zeta(s) \zeta(s-1) \zeta(s-2)$ \\
\cline { 2 - 5 } & $\widehat{c}_{H, G}$ & & $3^{-s}\left(1-2^{-s}\right) \zeta(s)^{2}\left(\left(1+3 \cdot 2^{-s}\right) \zeta(s-1)+2 \vartheta(s)\right)$ \\
\hline \multirow{2}{*}{$\mathcal{G}_{3}$} & $\widehat{s}_{H, G}$ & $\left(1-3^{-s}\right) \zeta(s-1)^{2} \vartheta(s-1)$ & $2^{-s}\left(1-3^{-s}\right) \zeta(s-1)^{2} \vartheta(s-1)$ \\
\cline { 2 - 5 } & $\widehat{c}_{H, G}$ & $\left(1-3^{-s}\right)\left(1+2 \cdot 3^{-s}\right) \zeta(s)^{2} \vartheta(s)$ & $2^{-s}\left(1-3^{-s}\right)\left(1+3^{-s}\right) \zeta(s)^{2} \vartheta(s)$ \\
\hline \multirow{2}{*}{$\mathcal{G}_{5}$} & $\widehat{s}_{H, G}$ & & $\left(1-2^{-s}\right)\left(1-3^{-s}\right) \zeta(s-1)^{2} \vartheta(s-1)$ \\
\cline { 2 - 4 } & $\widehat{c}_{H, G}$ & & $\left(1-2^{-s}\right)\left(1-3^{-s}\right) \zeta(s)^{2} \vartheta(s)$ \\
\hline
\end{tabular}

\section{References}

[1] Chelnokov G., Deryagina M., Mednykh A. (2017) . On the coverings of Euclidean manifolds $\mathbb{B}_{1}$ and $\mathbb{B}_{2}$, Comm. Algebra 45 (4): 1558-1576.

[2] Chelnokov G., Mednykh A. (2017) On the coverings of Euclidean manifolds $\mathbb{G}_{2}$ and $\mathbb{G}_{4}$. preprint https://arxiv.org/pdf/1805.08146.pdf.

[3] Conway J. H., Rossetti J. P. (2003). Describing the Platycosms // arXiv:math.DG/0311476v1.

[4] The Bilbao Crystallographic Server (http://www.cryst.ehu.es/cryst/cellsub.html)

[5] On-Line Encyclopedia of Integer Sequences, http://oeis.org/

[6] J. H. Conway and N. J. A. Sloane, "Sphere Packings, Lattices and Groups", SpringerVerlag

[7] Hatcher A. (2002), Algebraic Topology. Cambridge: Cambridge University Press.

[8] Hurwitz A. (1891). Über Riemann'sche Flächen mit gegebenen Verzweigungspunkten. Math. Ann., 39: 1-60. 
[9] Hurwitz A. (1902). Über die Anzahl der Riemann'schen Flächen mit gegebenen Verzweigungspunkten,. Math. Ann., 55: 53-66.

[10] Kwak J. H., Lee J., Mednykh A. D. (2008). Coverings, enumeration and Hurwitz problems, J. Koolen (ed.) et al., Applications of group theory to combinatorics. Selected papers from the Com2MaC conference, Pohang, Korea, July 9-12, 2007. Boca Raton, FL: CRC Press. 71-107.

[11] Liskovets V., Mednykh A. (2000). Enumeration of subgroups in the fundamental groups of orientable circle bundles over surfaces, Commun. Algebra 28 (4): 17171738 .

[12] Liskovets V., Mednykh A. (2000). The number of subgroups in the fundamental groups of some non-oriented 3-manifolds, in: Formal Power Series and Algebraic Combinatorics, Proceedings of the 12th International Conference, FPSAC'00, Moscow, Russia, June 26-30, 2000, Berlin: Springer, 276-287.

[13] Lubotzky A., Segal D. (2003). Subgroup Growth. Basel: Birkhäuser.

[14] Mednykh A. D. (1978). Determination of the number of nonequivalent coverings over a compact Riemann surface. Soviet Math. Dokl. 19(2): 318-320.

[15] Mednykh A. D. (1979). On unramified coverings of compact Riemann surfaces. Soviet Math. Dokl., 20(1): 85-88.

[16] Mednykh A. D. (1984). Nonequivalent coverings of Riemann surfaces with a prescribed ramification type, Siberian Math. J., 25(4): 606-625.

[17] Mednykh A. D. and Pozdnyakova, G. G. (1986). Number of nonequivalent coverings over a nonorientable compact surface. Siber. Math. J., 27(1): 99-106.

[18] Mednykh A. (2008). Counting conjugacy classes of subgroups in a finitely generated group. Journal of Algebra, 320(6): 2209-2217.

[19] Müller T.W. (2000). Enumerating representations in finite wreath products, Adv. Math. 153 (1): 118-154.

[20] Müller T.W., Shareshian, J. (2002). Enumerating representations in finite wreath products. II: Explicit formulae, Adv. Math. 171 (2): 276-331.

[21] Müller T.W., Puchta, J.-C. (2002). Character theory of symmetric groups and subgroup growth of surface groups, J. London Math. Soc. (2) 66: 623-640.

[22] Rattcliffe J. G., Tschantz S. T. (2009). Abelianization of space groups, Acta Cryst. A65: 18-27.

[23] Weisstein, Eric W. "Dirichlet Generating Function." From MathWorld-A Wolfram Web Resource. http://mathworld.wolfram.com/DirichletGeneratingFunction.html

[24] Weisstein, Eric W. "Perron's Formula." From MathWorld-A Wolfram Web Resource. http://mathworld.wolfram.com/PerronsFormula.html

[25] Wolf J. A. (1972). Spaces of Constant Curvature, New York: Mc Graw-Hill Book Company. 